\title{
Accurate Sheet Resistivity Measurement Based on Image Reconstruction Using Improved Node-back-projection Algorithm
}

\author{
Xinfu Liu, ${ }^{1}$ Qianwen Li, ${ }^{1}$ Hsiung-Cheng Lin, ${ }^{2 *}$ Pengfei Wu, ${ }^{1}$ and Mengdan Wang ${ }^{1}$ \\ ${ }^{1}$ School of Mechanical Engineering, Hebei University of Technology, Tianjin, China 300130 \\ ${ }^{2}$ Department of Electronic Engineering, National Chin-Yi University of Technology, Taichung, Taiwan 41170
}

(Received July 10, 2019; accepted August 22, 2019)

Keywords: pseudo-measurement method, sheet resistivity, electrical impedance tomography, node-backprojection algorithm

Currently, electrical impedance tomography (EIT) is widely used in sheet resistivity measurement of silicon wafers. The reconstruction of a resistivity image by electrical impedance imaging usually uses a four-probe measurement method. However, it may cause some loss of data and result in inaccurate outcomes. To resolve this problem, in this paper, we propose an improved node-back-projection algorithm by integrating the pseudomeasurement principle. High-resolution resistivity images can therefore be obtained quickly without damaging the silicon wafer. The resistivity contour map is then formed to identify the resistivity distribution. Accordingly, the positions of defects in the silicon wafer can be determined from the measured image, making the quality of silicon-wafer-based chips more reliable. The experimental results verify that the proposed algorithm is superior in terms of precision, reliability, and speed.

\section{Introduction}

The integrated circuits produced from single-crystal silicon wafers are becoming increasingly compact. As a result, the determination of the back-side contact resistance of semiconductor wafers from surface potential measurements has become more important. As reported in a previous paper, ${ }^{(1)}$ the potential distribution on the front surface of a wafer can be used for the effective monitoring of the back-side contact resistance because of its strong dependence on the contact resistance. The uniformity of the resistivity distribution of a silicon wafer may greatly affect the reliability of semiconductor devices. There are many test methods for the microscale-region resistivity of a silicon wafer in industry, e.g., the extended resistance probe method, two-probe method, three-probe method, four-probe method, schottky junction probe method, eddy current method, ${ }^{(2)}$ plasma resonance infrared method, ${ }^{(3)}$ and microwave scanning microscope probe test method. Among them, the four-probe method is the most widely used resistivity measurement method in the semiconductor production process. The 
four-probe method ${ }^{(4,5)}$ is a simple means of measuring small samples and analyzing their inhomogeneity. However, the position of the probe requires manual adjustment. Also, the silicon wafer is easily contaminated because of the undesirable contact between the probe tip and the silicon wafer surface. This may cause inaccurate measurement results. A three-probe method with less complexity for quickly measuring the resistivity of silicon wafers was reported. ${ }^{(6)}$ However, the problem of silicon wafer contamination is still unsolved. A photoluminescence method $^{(7)}$ was proposed to reduce the contamination and detect silicon defects from the resistivity distribution of a silicon wafer. Unfortunately, the results cannot be interpreted intuitively so it is not easy to apply such a method in industry.

To overcome the drawbacks of the above methods, electrical impedance tomography (EIT), ${ }^{(8)}$ which is a noninvasive image reconstruction method, was proposed to measure the sheet resistance of silicon wafers. This technology includes two aspects solving the positive and the inverse problem. ${ }^{(9)}$ The inverse problem of EIT is mainly the process of calculating the resistivity distribution from the potential distribution. Well-known methods used to deal with negative aspects include the filtered back-projection method, ${ }^{(10)}$ projection error propagationbased regularization (PEPR) method, ${ }^{(11)}$ radial basis neural network, Gauss-Newton method, ${ }^{(12)}$ expectation maximum (EM) method, ${ }^{(13)}$ and particle swarm optimization. ${ }^{(14)}$ These methods were classified into three categories by Rensselaer Polytechnic Institute as those for solving linear inverse problems, iterative methods for solving nonlinear inverse problems, ${ }^{(15)}$ and methods for directly solving inverse problems.

The finite element method (FEM) is used to deal with positive aspects. ${ }^{(16)}$ However, the huge number of parameters associated with the FEM model makes EIT reconstructions neither stable nor computationally efficient. Alternatively, the node-back-projection algorithm ${ }^{(17)}$ and the pseudo-measurement method can be combined to reconstruct a resistivity image. ${ }^{(18)}$ The resistivity image of a silicon wafer can therefore be effectively reconstructed with a small amount of known data. This combined method can also reduce the contamination of the silicon wafer, increase the measurement speed, and improve the measurement accuracy. With the continued increase in the width of silicon wafers and the shrinking of circuit dimensions, the measurement of the sheet resistance is becoming increasingly stringent, especially in the solar cell, microelectronics reliability, and electrical component industries. ${ }^{(19-21)}$ This paper is divided into three parts. The first part presents the basic theory of the node-back-projection algorithm and the improved pseudo-measurement method. The second part describes the structure of the hardware circuit. The third part shows a comparative analysis of experimental results.

\section{Improved Node-back-projection Algorithm}

\subsection{Review of principle of node-back-projection algorithm}

There are various back-projection algorithms reported in the literature. ${ }^{(22)}$ Generally, 16 electrodes on the wafer boundary are equally placed and numbered separately, as shown in Fig. 1. The distribution of the potential on the boundary is continuous and regular. The potential 


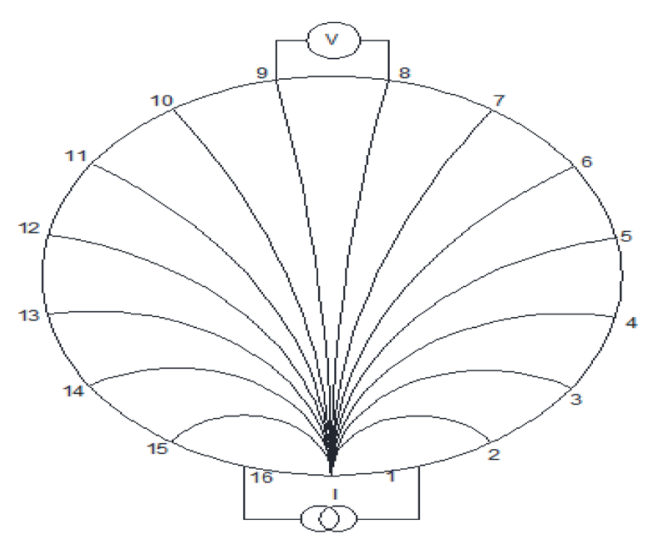

Fig. 1. Silicon wafer equipotential line distribution.

of each electrode of a standard silicon wafer under a certain excitation is measured by curve fitting. Then, the voltage between the electrodes is measured by applying the same excitation to the test silicon wafer. As a result, the distribution of resistivity in the silicon wafer is obtained.

The physical field model is established from Fig. 1. The conductivity $\sigma$ has the following relationship with the scalar potential function $\varphi$ and the current $J$ :

$$
\sigma=-\frac{J}{\nabla \varphi}
$$

The above formula can be converted into a resistivity form as

$$
\rho=-J^{-1} \nabla \varphi
$$

The current $J$ is constant. Accordingly, it can be seen that the resistivity is proportional to the potential gradient. When the resistivity $\rho_{0}$ changes to $\rho_{1}$, the potential $\varphi_{0}$ changes to $\varphi_{1}$. The following relationship is thus established:

$$
\Delta \rho=\rho_{1}-\rho_{0}=-J^{-1}\left(\nabla \varphi_{1}-\nabla \varphi_{0}\right)=-J^{-1} \nabla\left(\varphi_{1}-\varphi_{0}\right) .
$$

According to the definition of back-projection, the projection line should be consistent with the potential line in the field, as shown in Fig. 2. If current is injected into adjacent electrodes, the current starts from one excitation electrode and ends at the adjacent electrode.

Under two-dimensional conditions, each potential line in the silicon wafer can be equivalent to a point when projected onto the boundary. Similarly, the two-dimensional resistive surface becomes the line resistance on the boundary after projection, as shown in Fig. 3.

This two-dimensional silicon wafer model is equivalent to the one-dimensional equivalent model circuit shown in Fig. 4. The $X$-direction represents the position of each equipotential line projected on the boundary. The $Y$-direction represents the measured voltage on the boundary. 


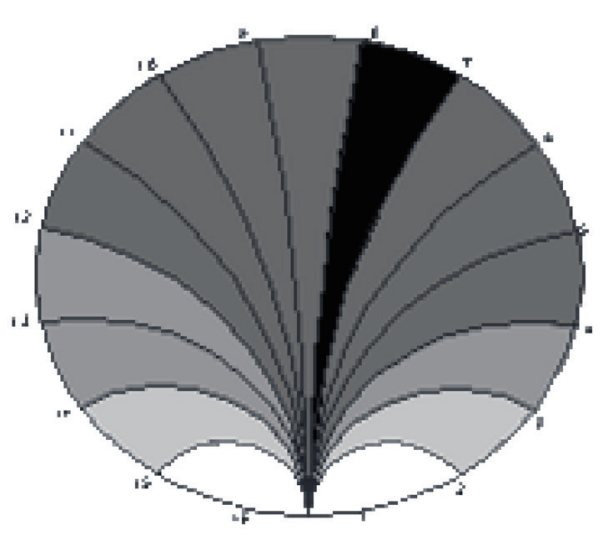

Fig. 2. Adjacent mode projection area using nodeback-projection method.

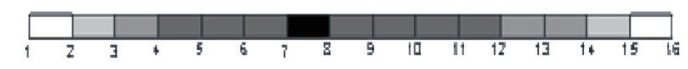

Fig. 3. Projected equivalent line resistance.

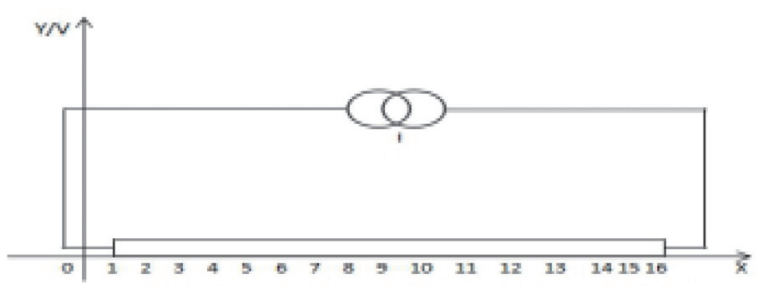

Fig. 4. One-dimensional current equivalent model circuit.

In the one-dimensional model above, the resistivity of each point on the line resistance is that of the equipotential line $\mathrm{L}$ projected to this point. The value is equal to the sum of the total resistivities at the point on the equipotential line $\mathrm{L}$ in the silicon wafer. It can be expressed by the following integral equation:

$$
\rho=\int \rho(L) d L
$$

As can be seen from Fig. 4, the relationship between the boundary potential function $\varphi(x)$ and the equivalent resistivity function $\rho(x)$ is

$$
\varphi(x)=\int J \rho(x) d x
$$

and

$$
\rho(x)=-J^{-1} \frac{d \varphi}{d x} .
$$

Equation (7) can be obtained from Eqs. (3) and (6).

$$
\Delta \rho=\frac{d\left(\varphi_{1}-\varphi_{0}\right)}{d x}
$$

As above, when the internal resistivity of the silicon wafer changes from the initial state $\rho_{0}$ to $\rho_{1}$, the dynamic change can be obtained from Eq. (7). The dynamic change is back-projected to the equidistant line in the mapped region in accordance with the projection relationship determined in the initial state. After measuring the interelectrode rotation excitation, the obtained data is superimposed to find the change in electrical impedance in the silicon region. By comparing with the initial state, we can obtain the real-time resistivity distribution in the silicon region. 


\subsection{Improved node-back-projection algorithm using pseudo-measurement method for obtaining boundary functions}

The pseudo-measurement method can fit a function to the boundary voltage data to improve the accuracy of the reconstructed image. The boundary voltage distribution of silicon wafers in an adjacent current excitation mode is continuous and monotonic. On the basis of limited measurement data, the boundary potential distribution function is obtained by function interpolation or a fitting method, which can improve the prior conditions of the reconstruction algorithm and increase the measurement information. Generally, it is difficult to obtain the exact expression for a potential distribution function directly by function fitting. Usually, a simple analytic function can be constructed and approximated by the least-squares method. ${ }^{(23)}$ Therefore, a power series polynomial can be used to construct an analytic function for use as the boundary voltage distribution function.

Assuming that the boundary potential distribution function in the initial state is $\varphi_{0}(x)$, the function $P(x)$ is constructed as follows to approximate to the function $\varphi_{0}(x)$.

$$
P(x)=p_{1} x^{n}+p_{2} x^{(n-1)}+\cdots+p_{n} x+p_{n+1} .
$$

where $n$ is the polynomial order, $x$ is a boundary position coordinate, and $p_{i}(i=1,2, \ldots, n+1)$ are coefficients.

The least-squares method is used to solve $\varphi_{0}(x)$. The problem is transformed into finding the minimum $\min \left[J\left(p_{1}, p_{2}, \ldots, p_{n+1}\right)\right]$, where

$$
J\left(p_{1}, p_{2}, \cdots, p_{n+1}\right)=\sum_{i=1}^{m} \delta_{i}^{2}
$$

Here, $\delta_{i}$ is the residual between the measured value $y_{i}$ at the boundary electrode and the predicted value $p\left(x_{i}\right)$ of the constructed function.

$$
\delta_{i}=p\left(x_{i}\right)-y_{i}=\sum_{j}^{n+1} p_{j}\left(x_{i}\right)^{n+1-j}-y_{i}
$$

When $\delta_{i}$ takes its minimum value, a unique set of coefficients can be obtained using the multivariate function to find the extremum of $p=\left[p_{1}, p_{2}, \ldots, p_{n+1}\right]$. The fitting function $\varphi_{0}(x)$ is obtained by substituting the coefficients into Eq. (8).

$$
\varphi_{0}(x)==\sum_{i=1}^{n+1} p_{i} x^{n+1-i}=p_{1} x^{n}+p_{2} x^{n-1}+\cdots+p_{n} x+p_{n+1}
$$

$\varphi_{0}(x)$ is used to calculate the potential at each position on the boundary so that the coordinates of the nodes in the silicon wafer corresponding to the boundary positions can be 
obtained. When the resistivity distribution in the silicon wafer changes, the boundary potential function changes from $\varphi_{0}(x)$ to $\varphi_{1}(x)$. The same method can be applied to $\varphi_{1}(x)$ as follows:

$$
\varphi_{1}(x)==\sum_{i=1}^{n+1} q_{i} x^{n+1-i}=q_{1} x^{n}+q_{2} x^{n-1}+\cdots+q_{n} x+q_{n+1},
$$

where $n$ is the polynomial order, $x$ is a boundary position coordinate, and $q_{i}=(i=1,2, \ldots, n+1)$ are coefficients.

Substituting Eqs. (11) and (12) into Eq. (9), we obtain

$$
\Delta \rho=\frac{d\left(\varphi_{1}(x)-\varphi_{0}(x)\right)}{d x}=\sum_{i=1}^{n}\left(q_{i}-p_{i}\right)(n-i+1) x^{n-i} .
$$

The absolute change in the resistivity of each node in the silicon wafer can be obtained from Eq. (13). In the initial state, the boundary position coordinates corresponding to each node in the silicon wafer are calculated. Each obtained boundary position coordinate is substituted into Eq. (13) to find the equivalent resistivity of the one-dimensional circuit projected by the equipotential line of the node. Then, the equivalent resistivity is back-projected along the equipotential line. The change in resistivity at each node is thus obtained. Finally, the actual change in resistivity at each node is obtained by weighting and superimposing the changes in the resistivity of each node.

\section{Setup of Silicon Resistivity Measurement System}

The hardware system used to implement the proposed model is composed of six parts: an excitation source module, a multichannel analog switch module, a voltage measurement module, a DSP28335 chip, a serial communication circuit, and a computer. A functional modular diagram of the system is shown in Fig. 5. Sixteen electrodes are evenly placed at the edge of the silicon wafer. ${ }^{(24)}$ These electrodes are connected to multiple analog switches so that the excitation source injection and voltage measurement electrodes can be selected. The measured voltage data is processed by a signal amplifier circuit and transmitted to the subsequent dataprocessing circuit. This data-processing circuit is based on the DSP chip. ${ }^{(25)}$ The measurement data is transmitted to the computer for signal processing through the serial communication module. The DSP command controls the multichannel analog switch for the excitation electrode and the voltage measurement probe. The above steps are repeated until the silicon chip boundary voltage distribution function is obtained.

During the measurement process, the silicon wafers with different resistances need to be injected with different currents. To obtain the optimal input current and improve the signal-tonoise ratio, the method of determining the optimal current ${ }^{(26)}$ and the optimal current excitation mode were proposed. ${ }^{(27,28)}$ As reported in a previous paper, ${ }^{(29)}$ an optimal current pattern and an optimal electric potential measurement strategy can improve the current density distribution 


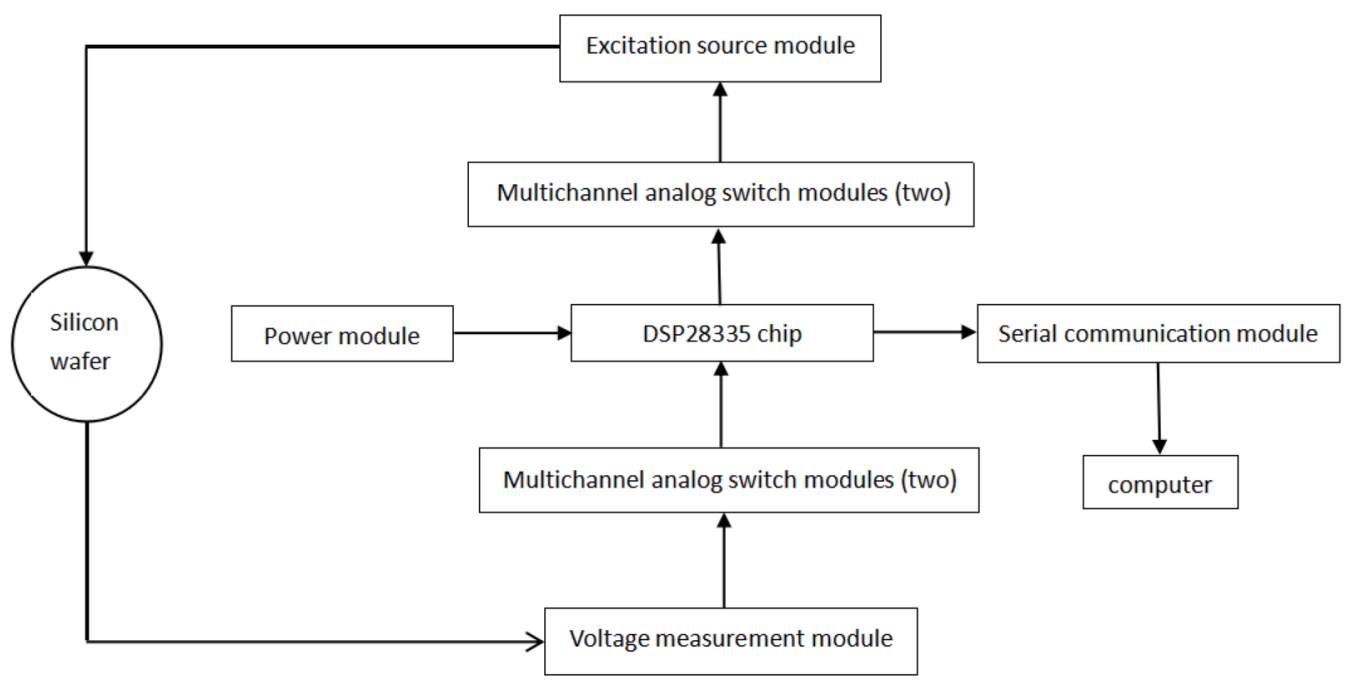

Fig. 5. Block diagram of hardware system for resistivity-measuring device.

and increase the convergence speed of the image reconstruction algorithm. In the case of an unknown resistivity distribution, however, the relationship between the excitation current and the boundary voltage cannot be accurately deduced in a practical situation. For this reason, the proposed programmable voltage-controlled constant current-source circuit is designed using a fuzzy control algorithm. ${ }^{(30)}$ The magnitude of the injection current is controlled by changing the power supply voltage. A schematic diagram of a three-operational-amplifier V/I conversion circuit is shown in Fig. 6.

When all three operational amplifiers are at an ideal working state, the output current can be obtained as

$$
I=\frac{V_{-} \frac{R_{3}+R_{4}}{R_{3}}\left[\frac{V_{+}-V}{R_{1}}\left(R_{1}+R_{2}\right)+V\right]}{R_{5}},
$$

where $V_{+}$is the non-inverting output voltage and $V_{-}$is the inverting output voltage.

According to the "virtual-short" characteristic of the ideal amplifier, $V_{+}$is equal to $V_{-}$. When $R_{1}=R_{4}=R_{2}=R_{3}$, the equation can be simplified to

$$
I=\frac{V}{R_{5}}
$$

Accordingly, the output current is proportional to the input voltage. The output current $I$ can thus be adjusted by the input voltage $V$. 


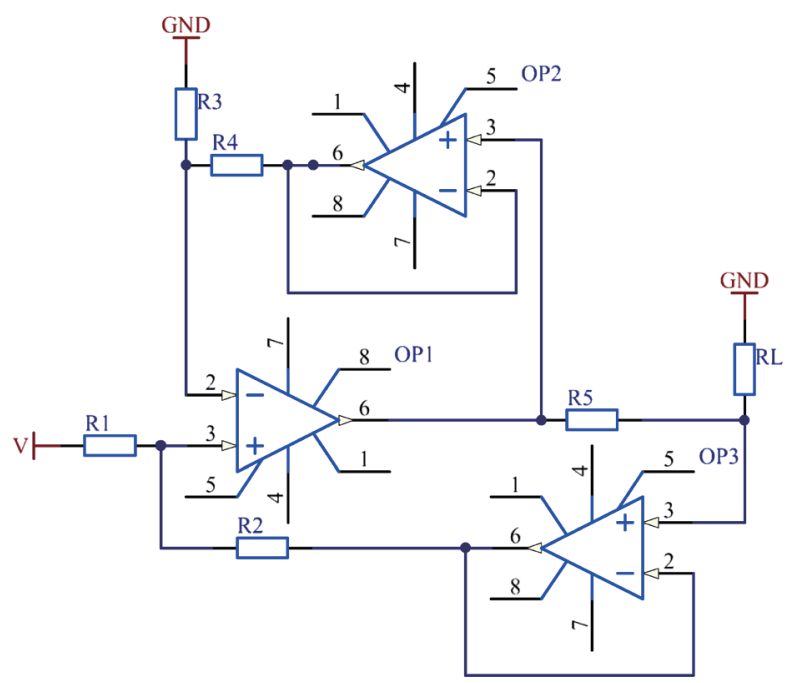

Fig. 6. (Color online) Schematic of three-operational-amplifier constant-current source circuit for pressure control.

The fuzzy controller is mainly divided into three parts with the following functions: (1) Blur the input quantity. (2) Output fuzzy variables by the fuzzy reasoning of fuzzification data. (3) Defuzzify the fuzzification outputs. ${ }^{(31)}$ A diagram showing the principle is given in Fig. 7.

The error $(e)$ and error rate $(e c)$ of the boundary voltage response are selected as the input of the fuzzy controller, and the current increment $(u)$ is used as the controlled amount. In fuzzification, the actual change interval of the input needs to be converted into the domain of the blurring sets of the corresponding linguistic variables using quantifying factors. These factors can be expressed as

$$
\begin{gathered}
K_{e}=\frac{n}{e_{\max }}, \\
K_{e c}=\frac{m}{e c_{\max }} .
\end{gathered}
$$

The fuzzy domain of the error $(e)$ is defined as $[-n, n]$, and the fuzzy domain of the error rate $(e c)$ is defined as $[-m, m]$. The fuzzy level classification is divided into seven fuzzy subsets: negative big (NB), negative medium (NM), negative small (NS), zero (ZO), positive small (PS), positive medium (PM), and positive large (PB). The process of transforming the input from the membership function to a membership vector is called fuzzification. The membership function selects a triangular or trapezoidal function. Choosing a fuzzy controller with a double input and a single output requires the summarization of 49 fuzzy control rules according to the accumulated experience. A query table of the rules is shown in Table 1.

By using the Mamdani reasoning method, we synthesize the membership degree of each fuzzy rule, and the fuzzy relation $R$ of the whole control system is obtained as

$$
R=R_{1} \vee R_{2} \vee R_{3} \vee \cdots \vee R_{49}
$$




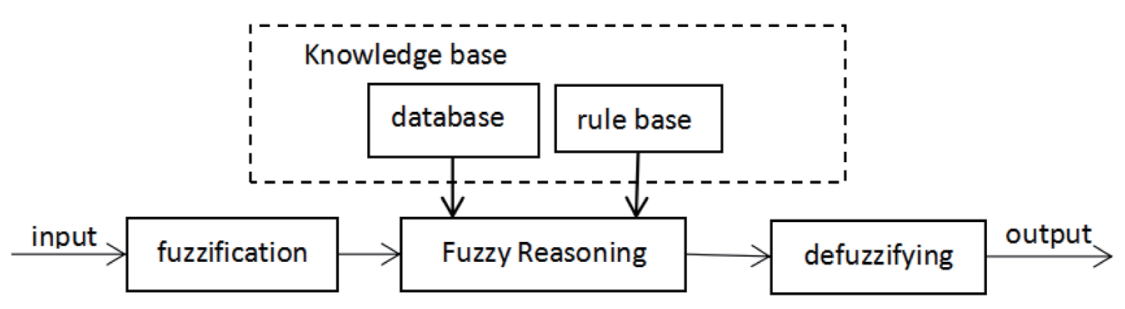

Fig. 7. Schematic of principle of fuzzy controller.

Table 1

Query table of fuzzy control rules.

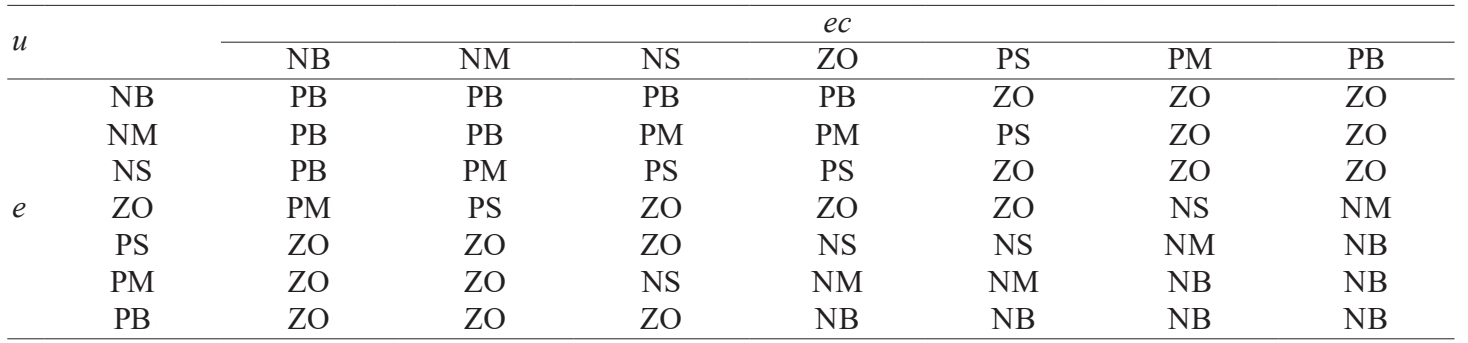

Defuzzification is the key step in the fuzzy controller used to control the system. It converts the output from a fuzzy value to an exact value by a mathematical method. The centroid method is used to solve the fuzzification. The value of the center point of the area of the fuzzy function is taken as an exact quantity, as shown in Eq. (19). The centroid method contains all the information on elements in the output fuzzy subset; thus, the obtained results are more accurate.

$$
e^{*}=\frac{\int \mu_{c}(e) e d e}{\int \mu_{c}(e) d e}
$$

The fuzzy control toolbox in MATLAB software is used. The FIS editor in the fuzzy control toolbox can be used to perform simulation experiments on the fuzzy controller, and the results are shown in Fig. 8.

As can be seen from Fig. 8, too low or too high current results in a large error in the voltage and error changing rate. Note that the current giving zero voltage error and error changing rate is the optimum solution. The theory of fuzzy reliability can also be used in many mechanical and electrical products. ${ }^{(32)}$

When an electrode in contact with a silicon wafer is used, a pressure of about $10 \mathrm{~N}$ is applied. During the placement of the electrode on the silicon wafer, repeated extrusions may result in the deformation of the soft material. Once the electrode is deformed, the contact area and pressure between the electrode and the silicon wafer will change during the contact process. This can cause the contact resistance to change greatly. Therefore, copper or copper alloy materials are usually selected for metal electrodes. Considering the fringe effect of the silicon wafer, the specific electrode arrangement is shown in Fig. 9. Figure 9(a) is the front view of electrodes in 


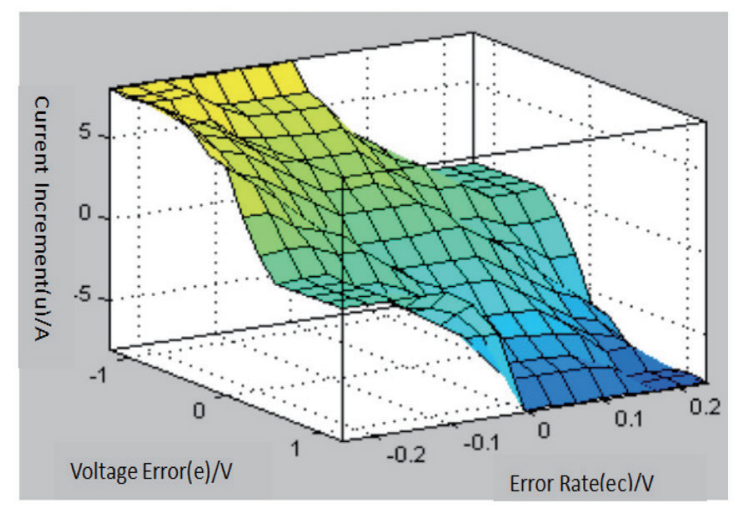

Fig. 8. (Color online) Surface map of fuzzy control input/output.

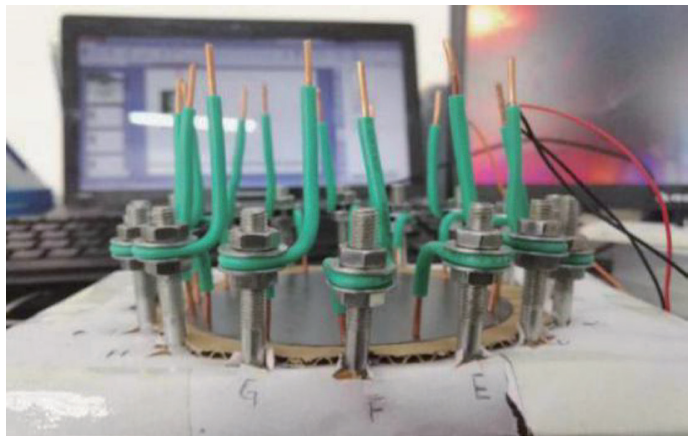

(a)

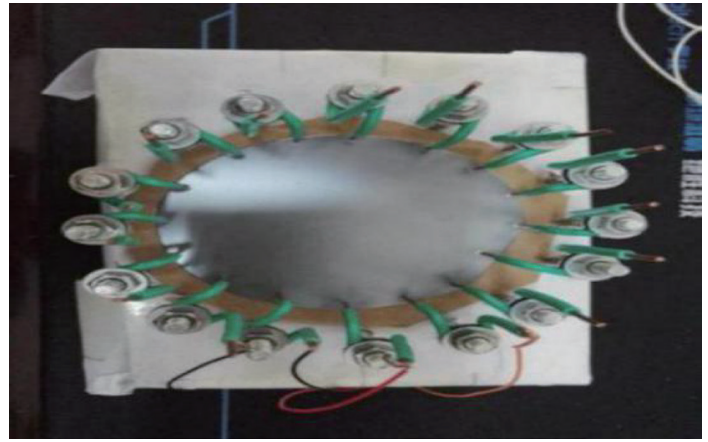

(b)

Fig. 9. (Color online) (a) Silicon boundary voltage tester and (b) position of electrode added to silicon wafer.

which the structure of a single electrode can be seen. Figure 9(b) is a top view of the electrode that is placed on the silicon wafer. Note that the upper part of the electrode is connected to the wire, and the electrode is placed on the silicon wafer near the edge.

\section{Experimental Results}

The silicon wafer used for measurement satisfies the following conditions: (1) The size of the wafer is 4 inches. (2) The crystal orientation is 100. (3) The test temperature of the environment is $25^{\circ} \mathrm{C}$. (4) The silicon resistivity ranges from 1 to $50 \Omega \cdot \mathrm{cm}$. (5) The surface is flat. (6) The probe forms an ohmic contact with the sample.

\subsection{Experiments using four-probe tester}

An RST.8-type four-probe tester whose resistivity ranges from $10^{-4}$ to $10^{5} \Omega \cdot \mathrm{cm}$ was used to measure the resistivity of the silicon wafer. When using the linear four-point probe method, 66 points on the silicon chip were selected to obtain definite data. From the coordinates of 
the measuring point, the position corresponding to each resistivity can be determined and the distribution of the silicon wafer resistivity can thus be obtained, as shown in Fig. 10. Generally, dark gray indicates a high resistivity and light gray represents a low resistivity. In this result, lightest gray corresponds to $30 \Omega \cdot \mathrm{cm}$ and darkest gray corresponds to $40 \Omega \cdot \mathrm{cm}$. An intuitive silicon resistivity mapping map can be obtained by converting the resistivity data into a grayscale image, as shown on the right-hand side of Fig. 10. During the measurement process, the position of each measuring point requires manual adjustment. Therefore, the entire test process is time-consuming and has low accuracy and resolution.

The measurement accuracy and resolution can be improved using a 4D-333A four-probe tester, with the same test method as the RST.8 four-probe tester. For a 4-inch and N-type silicon wafer, the resistivity isoline distribution is shown in Fig. 11 using different colors, and it can be displayed directly on a computer.

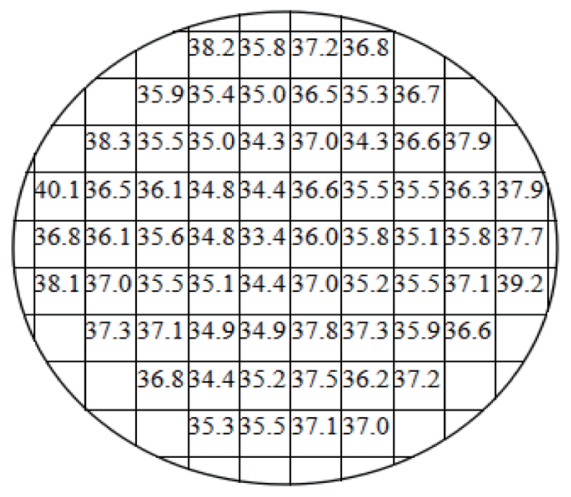

(a)

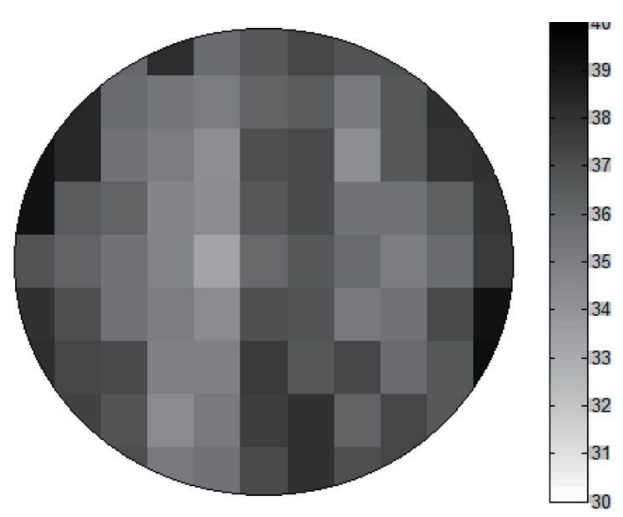

(b)

Fig. 10. (a) Map of silicon resistivity distribution and (b) mapping map (unit: $\Omega \cdot \mathrm{cm}$ ).

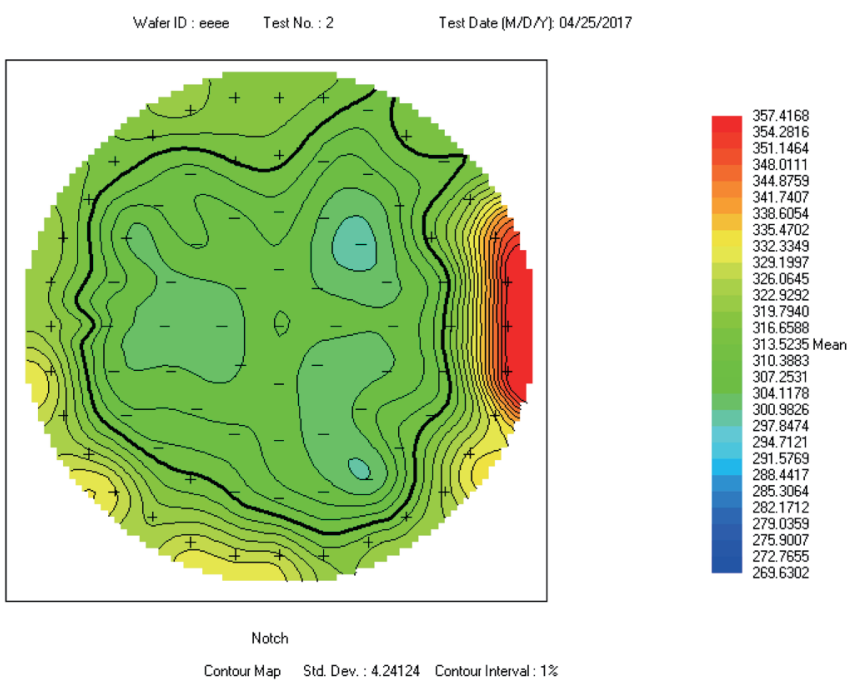

Fig. 11. (Color online) Wafer resistivity contour map (Unit: $\Omega / \square$ ). 


\subsection{Experiments using improved node-back-projection method}

\subsubsection{Fitting the boundary voltage function}

A total of 16 electrodes numbered 1 to 16 with the adjacent excitation mode are adopted to obtain the boundary voltage function. For example, electrodes 1 and 16 are selected as excitation electrodes and the remaining electrodes are used for measuring the voltage data. According to the principle of the pseudo-measurement method, using the polynomial curvefitting program of MATLAB to fit the 16 scatter voltages. Generally, the higher degree the polynomial fits, the closer the fitted curve is to the actual voltage distribution curve. To achieve a better fitting effect, we first select three and five polynomial fittings to test the performance. The result of five polynomial fittings is found to be much better than that of three polynomial fittings. The scattered points (UVS.X) of the boundary voltage obtained using the five-curvefitting function diagram are shown in Fig. 12. It can be seen that the boundary voltage near the excitation source has a large response. On the other hand, the response is small for the electrodes located away from the excitation source.

To further improve the accuracy of the voltage data, the result obtained using the six-curvefitting function is shown in Fig. 13. It is obvious that the curve can better approximately fit the silicon boundary voltage function than five curve fittings. Accordingly, the fitting function is applied six times to perform the subsequent calculations to consider the complexity of the algorithm.

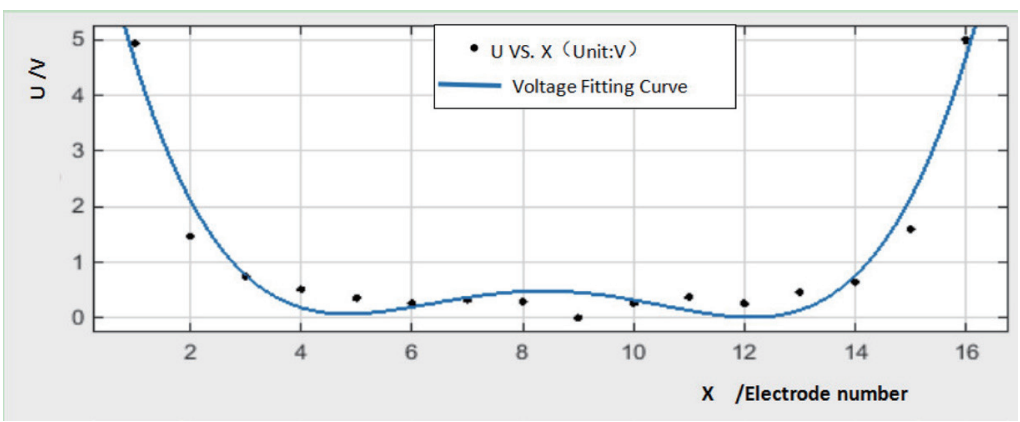

Fig. 12. (Color online) Five-curve-fitting function diagram of boundary voltage.

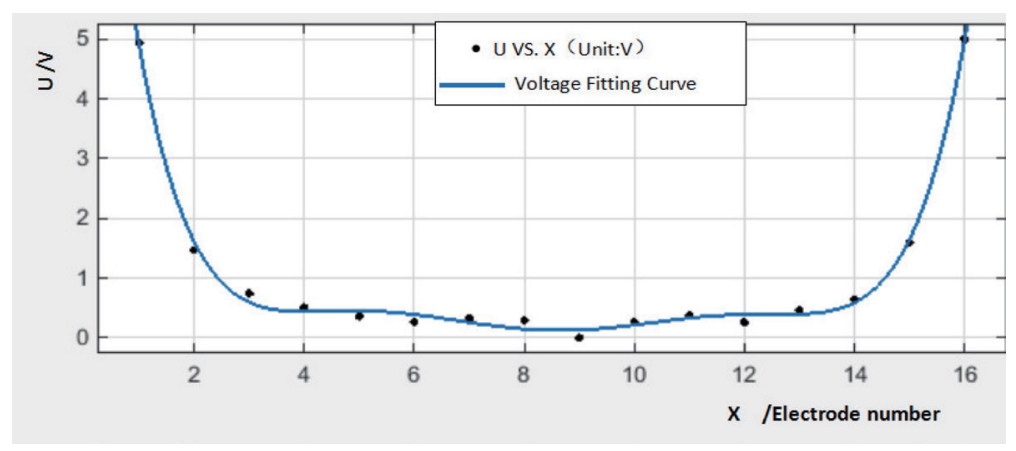

Fig. 13. (Color online) Six-curve-fitting function diagram of boundary voltage. 


\subsubsection{Reconstruction of image of silicon wafer resistivity}

In the node-back-projection algorithm, there are 16 rounds of excitation applied to the silicon wafer through the multichannel analog switch module. In other words, the silicon wafer is subject to sixteen excitations. The voltage data at the boundary of the silicon wafer is collected and transmitted to the computer. Therefore, the image of the silicon resistivity distribution is reconstructed, as shown in Fig. 14. Note that the information directly obtained from this algorithm is limited without using the silicon wafer boundary voltage function. For this reason, the resolution of the reconstructed resistivity image is still low.

Using the silicon wafer boundary voltage distribution function obtained by analyzing the boundary voltage data, the effectiveness of the node-back-projection algorithm in reconstructing the resistivity distribution of the silicon wafer can be improved by incorporating the pseudomeasurement method, as shown in Fig. 15. It can be seen that the resistivity of the central region in the silicon wafer is low, but that of the peripheral region is relatively high. However, the overall resistivity distribution is relatively uniform. The improved node-back-projection algorithm makes the image more accurate than that obtained using the RST.8 four-probe instrument. An intuitive image can be directly obtained without drawing the image separately.

The grayscale in Fig. 15 can be transformed into a contour map, as shown in Fig. 16, where the blue color depicts a low resistivity and the yellow color represents a high resistivity. Clearly, the map obtained using the proposed algorithm is more accurate than the wafer resistivity contour map shown in Fig. 11.

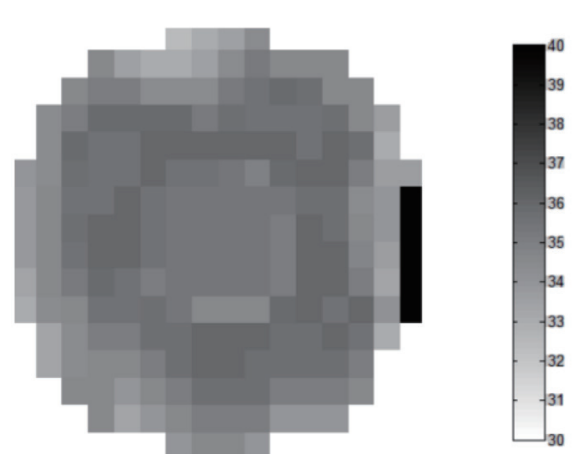

Fig. 14. Image reconstructed using node-backprojection algorithm (unit: $\Omega \cdot \mathrm{cm}$ ).

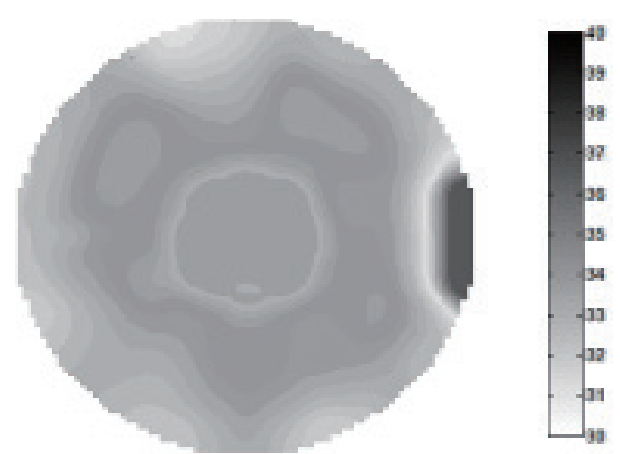

Fig. 15. Image reconstructed using improved nodeback-projection algorithm (unit: $\Omega \cdot \mathrm{cm}$ ).

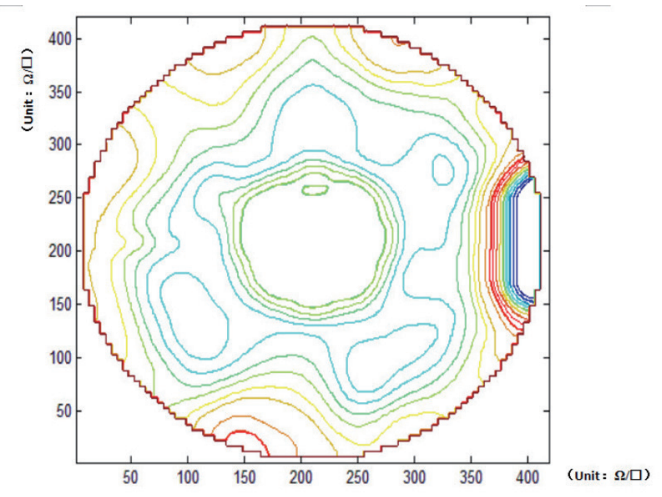

Fig. 16. (Color online) Silicon resistivity contour map. 


\section{Conclusions}

Generally, the four-probe method is the most widely used resistivity measurement method in a semiconductor production process. Unfortunately, it may suffer from some deficiencies such as inaccuracy and contamination. Although the contamination of a silicon wafer can be reduced using the node-back-projection algorithm, the measurement accuracy is very sensitive to the amount of measured data. To increase the amount of data to overcome this deficiency, the proposed algorithm has been successfully integrated with the pseudo-measurement method. Therefore, the resolution of the measurement is increased and the resistivity distribution map becomes clearer. Consequently, the positions of defects in a silicon wafer can be determined more effectively from the image of the measured resistivity. It has been proved by analyzing the experimental results that the resistivity distribution is consistent with that obtained with standard instruments. The accurate resistivity image of silicon wafers can be applied to solar panels in the energy industry.

\section{Acknowledgments}

The work was supported by the High Level Talented Person Funded Project of Hebei Province of China. It was also supported by the Excellent Experts for Going Abroad Training Program of Hebei Province of China.

\section{Conflicts of Interest}

The authors declare no conflicts of interest. The funders had no role in the design of the study; in the collection, analyses, or interpretation of data; in the writing of the manuscript; or in the decision to publish the results.

\section{References}

1 L. S. Tan, M. S. Leong, and S. C. Choo: Solid State Electron. 42 (1998) 589.

2 H. Wakiwaka, M. Kodani, M. Endo, and Y. Takahashi: Sens. Actuators A 129 (2006) 235.

3 V. Yashunsky, S. Shimron, V. Lirtsman, A. M. Weiss, N. Melamed-Book, M. Golosovsky, and B. Aroeti: Biophys. J. 97 (2009) 1003.

4 A. Q. Nguyen, G. Klysz, F. Deby, and J. Balayssac: Cem. Concr. Compos. 83 (2017) 308.

5 P. K. Rawat and B. Paul: Measurement 91 (2016) 613.

6 S. V. Lozanova and C. S. Roumenin: Measurement 43 (2010) 385.

7 D. Baek, J. Lee and B. Choi: Mater. Res. Bull. 58 (2014) 157.

8 H. C. N. Jongschaap, R. Wytch, J. M. S. Hutchison, and V. Kulkarni: Eur. J. Radiol. 18 (1994) 165.

9 H. Hakula, N. Hyvönen, and T. Tuominen: J. Comput. Appl. Math. 236 (2012) 4645.

10 G. A. P. Cirrone, M. Bucciolini, M. Bruzzi, G. Candiano, C. Civinini, G. Cuttone, and C. Talamonti: Nucl. Instrum. Methods Phys. Res., Sect. A 658 (2011) 78.

11 T. K. Bera, S. K. Biswas, K. Rajan, and J. Nagaraju: Measurement 49 (2014) 329.

12 J. F. Bao, C. Li, W. P. Shen, J. C. Yao, and S. M. Guu: Appl. Numer. Math. 111 (2017) 92.

13 Q. Wang, H. Wang, Z. Cui, and C. Yang: ISA Trans. 51 (2012) 808.

14 F. Marini and B. Walczak: Chemom. Intell. Lab. Syst. 149 (2015) 153.

15 M. Kumar, A. K. Singh, and A. Srivastava: J. Egypt. Math. Soc. 21 (2013) 334.

16 K. E. Akdogan, A.Yilmaz, and B. Saka: Proc. 2003 IEEE 46th Midwest Symp. Circuits and Systems (IEEE, 2003) 356 . 
17 M. Z. Haque: Comput. Biol. Med. 21 (1991) 289.

18 A. J. A. Simões Costa and M. Tardio Arze: Int. J. Electr. Power Energy Syst. 18 (1996) 73.

19 L. L. Li, X. B. Zhang, M. L. Tseng, M. Lim, and Y. Han: J. Cleaner Pro. 185 (2018) 198.

20 M. C. López-Escalante, F. Martín, and J. Ramos-Barrado: Sol. Energy 131 (2016) 61.

21 L. Li, Y. Xu, Z. Li, P. Wang, and B. Wang: Micro. Relia. 66 (2016) 58.

22 X. Yang, R. Yang, Y. Zhang, and D. Zhou: Neurocomputing 162 (2015) 171.

23 X. Wan, Y. Li, C. Xia, M. Wu, J. Liang, and N. Wang: Measurement 86 (2016) 93.

24 D. Chakraborty, M. Chattopadhyay, and R. Bhar: Procedia Technol. 10 (2013) 191.

25 M. K. Masten and I. Panahi: Control Eng. Pract. 5 (1997) 449.

26 P. Hua, E. J. Woo, J. G. Webster, and W. J. Tompkins: Proc.1992 IEEE Trans. Medical Imaging (IEEE, 1992) 488.

27 E. Demidenko, A. Hartov, N. Soni, and K. D. Paulsen: IEEE Trans. Biomed. Eng. 52 (2005) 238.

28 J. P. Kaipio, A. Seppänen, A. Voutilainen, and H. Haario: Inverse Prob. 23 (2007) 1201.

29 O. L. Silva, R. G. Lima, T. C. Martins, F. S. Moura, R. S. Tavares, and M. S. G. Tsuzuki: Control Eng. Pract. 58 (2017) 276.

30 K. H. Chua, Y. S. Lim, and S. Morris: Energy 122 (2017) 265.

31 G. Feng: Proc. 2006 IEEE Trans. Fuzzy Systems (IEEE, 2006) 676.

32 L. L. Li, C. M. Lv, M. L. Tseng, and J. Sun: Appl. Soft Comput. 65 (2018) 69. 